\title{
Robotic-assisted esophageal diverticulectomy
}

\author{
Graeme Rosenberg ${ }^{1}$, Mark F. Berry ${ }^{2}$ \\ ${ }^{1}$ Department of Surgery, ${ }^{2}$ Department of Cardiothoracic Surgery, Stanford University, Stanford, CA, USA \\ Contributions: (I) Conception and design: Both authors; (II) Administrative support: Both authors; (III) Provision of study materials or patients: \\ Both authors; (IV) Collection and assembly of data: Both authors; (V) Data analysis and interpretation: Both authors; (VI) Manuscript writing: Both \\ authors; (VII) Final approval of manuscript: Both authors. \\ Correspondence to: Mark F. Berry, MD. Mylavarapu Rogers Professor, Department of Cardiothoracic Surgery, Stanford University, 300 Quarry Drive, \\ Falk Cardiovascular Research Building, $2^{\text {nd }}$ Floor, Stanford, CA 94305, USA. Email: berry037@stanford.edu.
}

\begin{abstract}
Esophageal diverticula are relatively uncommon and can occur along any portion of the esophagus. Distal esophageal diverticula generally occur in the setting of motility disorders but can be found in patients who otherwise have normal esophageal motility. Larger esophageal diverticula are generally symptomatic, and intervention is typically indicated. Surgical management of mid or distal esophageal diverticulum was traditionally done via a thoracotomy, but minimally invasive surgery via thoracoscopy or laparoscopy have more recently been shown to be feasible and safe. We present a case here of a roboticassisted thoracoscopic approach to management of a large mid-esophageal diverticulum.
\end{abstract}

Keywords: Esophageal dysmotility; esophageal diverticulum; minimally invasive esophageal surgery

Received: 08 April 2020; Accepted: 21 July 2020; Published: 20 April 2021.

doi: 10.21037/jovs-2019-27

View this article at: http://dx.doi.org/10.21037/jovs-2019-27

\section{Introduction}

Esophageal diverticula can occur along any portion of the esophagus and are typically described by their anatomic location or wall structure (1). True diverticula occur in the mid-esophagus, include all structures of the esophageal wall, and are generally due to traction on the esophageal wall secondary to inflammation of adjacent mediastinal lymph nodes. These diverticula are most commonly associated with granulomatous disease of hilar or mediastinal lymph nodes due to sarcoidosis, tuberculosis, or histoplasmosis. Diverticula in the proximal (pharyngoesophageal or Zenker's diverticula) or distal $10 \mathrm{~cm}$ of the esophagus (epiphrenic) are false or pulsion diverticula that do not involve all layers of the esophageal wall $(2,3)$. These diverticula generally are composed of mucosa and submucosa, possibly with an overlying attenuated muscle layer. Zenker's diverticula (Figure 1) are felt to result from impaired relaxation of the cricopharyngeus muscle that leads to increased intraluminal pressure proximal to the upper esophageal sphincter. Distal esophageal diverticula (Figure 2) generally occur in the setting of motility disorders such as achalasia or diffuse esophageal spasm, though many can occur with apparently normal esophageal motility.

Asymptomatic patients with diverticula smaller than $5 \mathrm{~cm}$ can be managed expectantly. Larger esophageal diverticula are generally symptomatic, and intervention is typically indicated (3). Zenker's diverticula historically were managed via an open cervical surgical approach, though endoscopic approaches with stapler-assisted diverticulostomy or carbon dioxide laser-assisted diverticulotomy are available and now typically preferred over open surgery when technically feasible (4). The current preferred approach to surgical management of mid or distal esophageal diverticula is to utilize minimally invasive techniques (5). Given the normal course of the thoracic esophagus, mid-esophageal diverticula are approached via the right chest (6). Epiphrenic diverticula can be intervened upon using a transhiatal $(7,8)$ or transthoracic approach via the left or right chest depending on location of the diverticulum $(5,9,10,11)$. In this manuscript, we present a case of a mid-esophageal diverticulum that was managed via robotic-assisted resection and repair through the right chest. 


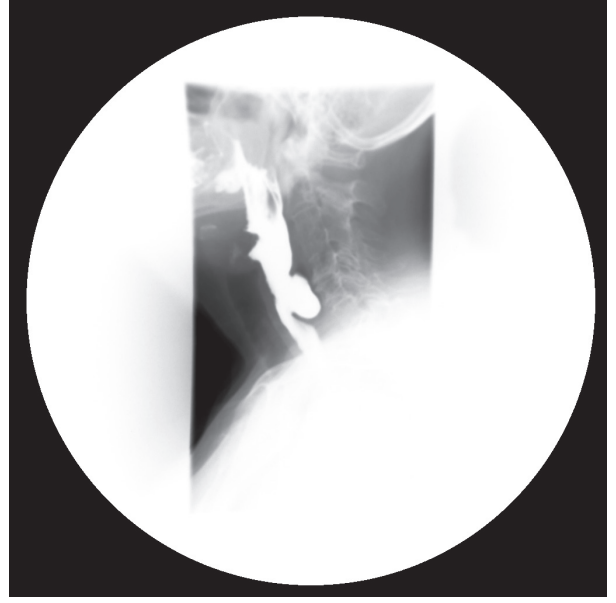

Figure 1 Barium swallow of a patient with a Zenker diverticula.
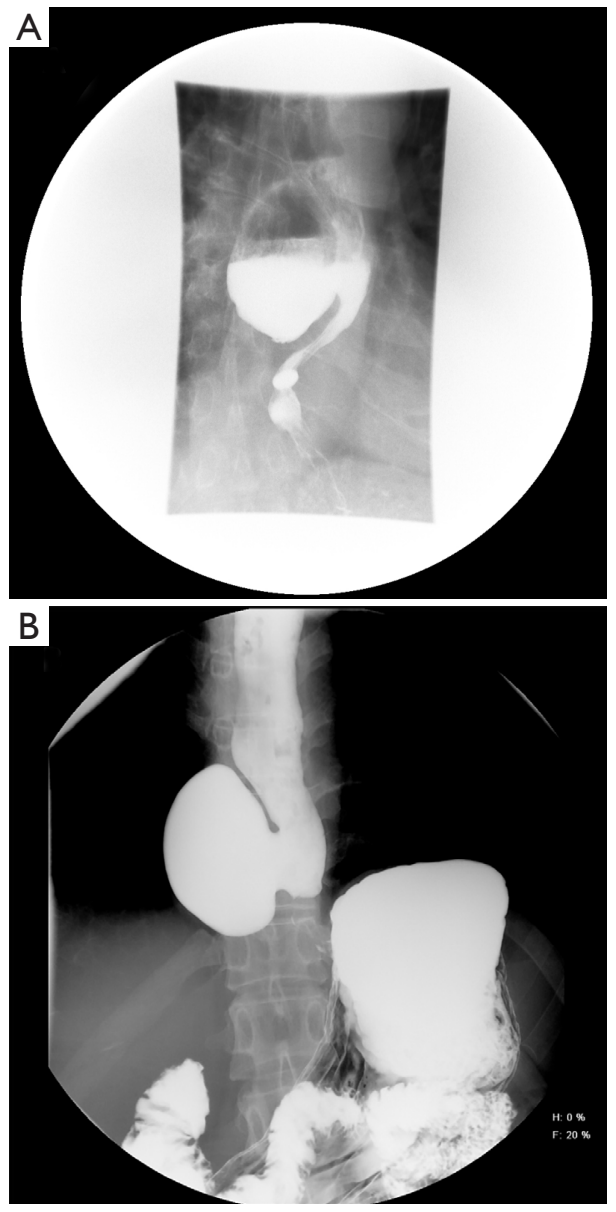

Figure 2 Barium swallows of patients with a diverticula in (A) the mid-distal esophagus and (B) the distal esophagus just above the gastro-esophageal junction.

\section{Patient presentation}

Many thoracic esophageal diverticula are asymptomatic and discovered incidentally during other workup for aerodigestive pathology. Asymptomatic esophageal diverticula can be managed conservatively by following patients clinically and with surveillance imaging and upper endoscopy if the patient develops any new signs or symptoms. However, symptomatic esophageal diverticula can diminish quality of life secondary to dysphagia, food impaction, regurgitation, and increased risk of aspiration pneumonia. Retrosternal pain and chronic cough may be present and halitosis is a common finding. Patient history may include chronic reflux, while patients with aspiration can have chronic pneumonia or even lung abscesses. In the case described here (Figure $2 A$ ), the patient had initially incidentally been found to have a diverticulum on routine upper endoscopy and did not develop symptoms due to food impaction until 6 years later.

\section{Workup}

Essentials of preoperative workup include establishing the size and location of diverticula. At a minimum, an esophagram and endoscopy are necessary not only to establish the diagnosis but also to evaluate the specific location of the diverticular opening, which is critical to guiding the approach for surgical management. The initial diagnostic workup often is the barium esophagram, which characterizes features of diverticula very well (Figure 2). In some situations, diverticula may have been initially discovered on cross sectioning imaging with CT scans (Figure 3), which can often clearly demonstrate the general location of a diverticulum. When a diverticulum is identified or suspected on a CT scan, we strongly suggest that patients proceed with a swallow study to clearly demonstrate the location and character of the diverticulum for surgical planning. Establishing location within the mid or distal thirds of the esophagus is paramount as diverticula more than $10 \mathrm{~cm}$ above the diaphragm are typically not amenable to a transhiatal approach and must be approached through the chest. Upper endoscopy is indicated when a diverticulum is found to exclude esophageal or gastric malignancy, other mucosal abnormalities, severe esophagitis, and Barrett's esophagus. When surgical resection is planned, endoscopy can be done immediately before surgical management if not done previously. Repeat endoscopy in 

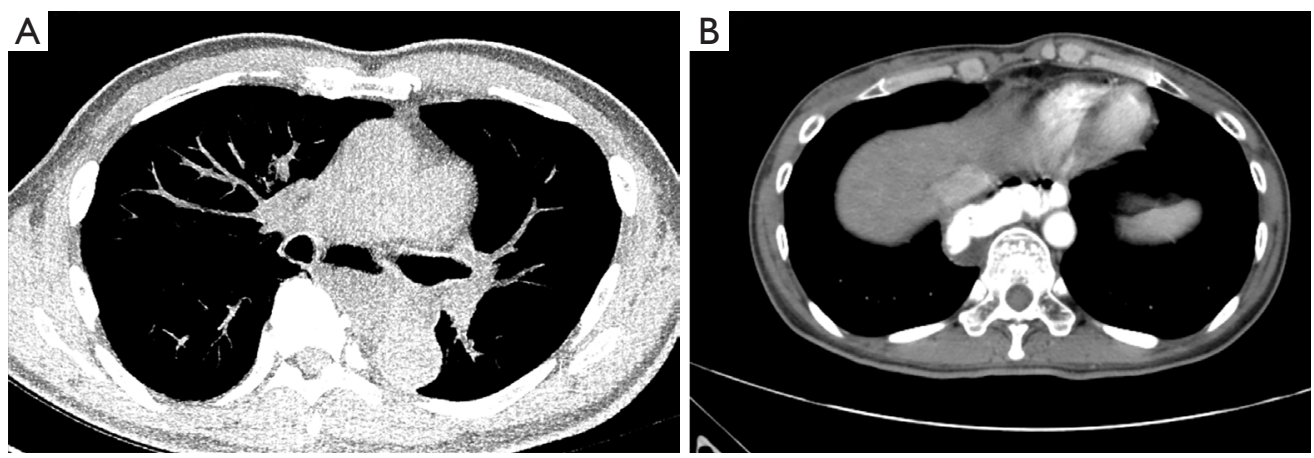

Figure 3 CT scans of patients with a diverticula in (A) the mid-distal esophagus and (B) the distal esophagus just above the gastroesophageal junction.
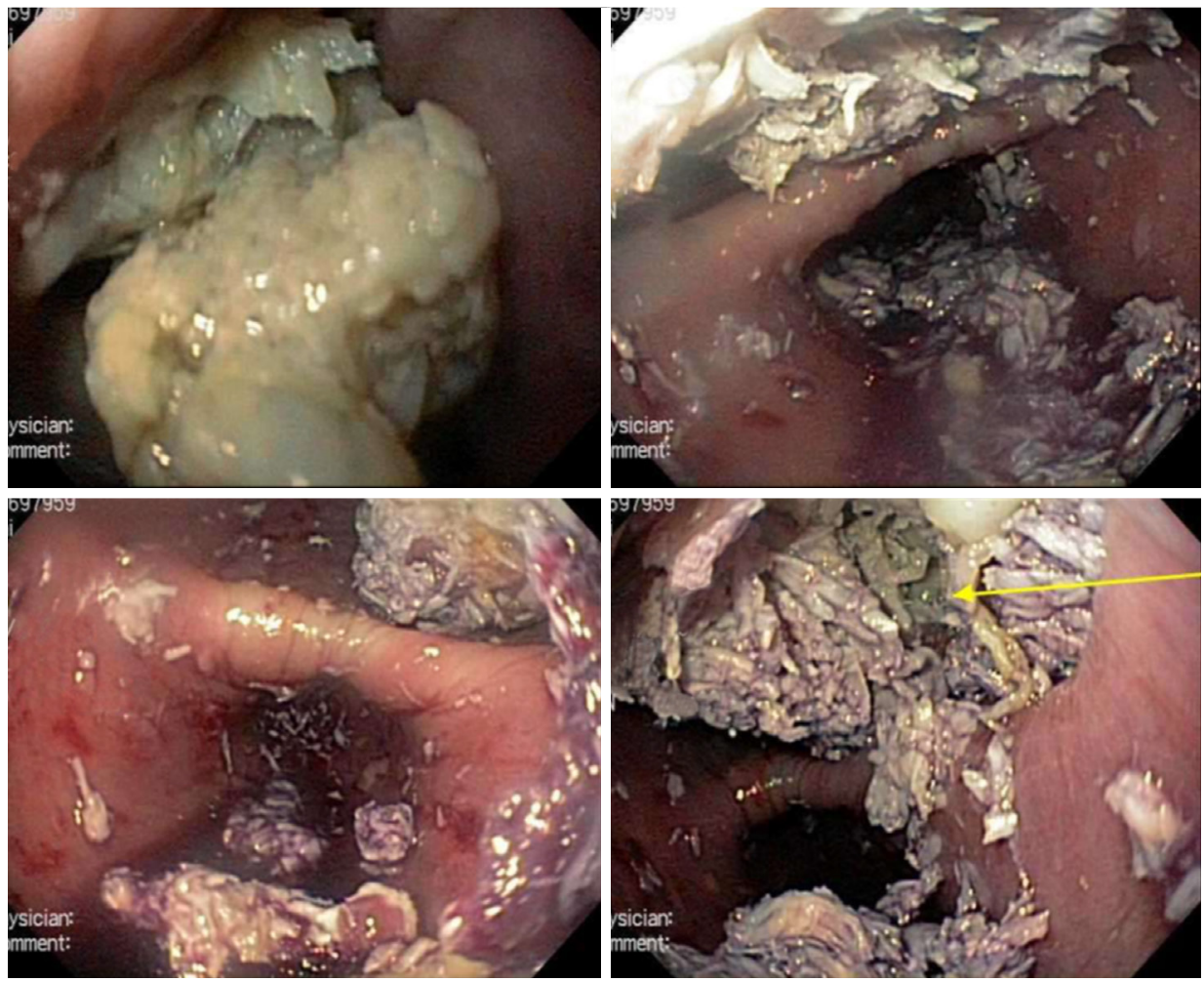

Figure 4 Pre-operative endoscopy of patient with a large mid-esophageal diverticulum, showing a wide-mouthed opening with a significant amount of debris in the diverticulum.

the immediate preoperative period even if already done is also strongly encouraged to remove impacted food from the diverticulum, which otherwise could influence surgical manipulation and resection of the diverticulum (Figure 4). Bronchoscopy can be considered in the evaluation of midesophageal diverticula if there is any suspicion of a tracheaesophageal fistula, if the diverticulum is adjacent to the airway on imaging, or if another underlying mediastinal process is suspected.

As discussed above, an underlying esophageal motility disorder is often present in patients with esophageal diverticula. Achalasia is frequently diagnosed. Alternative esophageal motility disorders that may be present include esophageal spasm, nutcracker esophagus, and hypertensive 
LES. Therefore, a motility evaluation is generally indicated if a patient does not already have a known diagnosis with esophageal manometry studies, ideally high-resolution manometry. Failure to diagnose and treat underlying esophageal motility disorders is known to increase recurrence rate and the risk of leak at the diverticular suture line. However, manometry is not always feasible as the presence of the diverticulum can make appropriate placement of the manometry probe problematic. In addition, current surgical dogma is to perform a long myotomy opposite and distal to the location of the diverticulum at the time of diverticulum resection regardless as to whether a known motility disorder is present or not, so manometry studies may not actually influence the surgical management of a diverticulum. Still, we recommend that manometry be attempted on all patients with large diverticula who are undergoing surgical management. At the least, identification of a motility abnormality can be helpful if a patient has or develops symptoms of dysphagia sometime after surgery, so to distinguish whether patients are having those symptoms secondary to surgery versus secondary to their underlying condition.

\section{Surgical management}

Surgical therapy for an esophageal diverticulum in the mid or distal esophagus involves resection of the diverticulum with diverticulectomy along with a long esophageal myotomy. Although there has been debate on the actual length of the myotomy that should be performed (5), we agree with the techniques reported in several series that the myotomy should extend from the upper limit of the diverticular neck to the gastroesophageal junction, though this can and should be modified based on a particular patient's motility. There has also been debate as to whether a fundoplication should be performed at the time of surgery (5). Most series on diverticulectomy describe the use of a concomitant fundoplication, though the specific type of wrap typically varies between surgeons. Given that the patients who require diverticulectomy have symptoms of dysphagia pre-operatively and likely have some underlying motility issue, we generally avoid a 360-degree Nissen fundoplication to minimize the risk of dysphagia due to the wrap unless the patient has a significant reflux history. Our general practice is to perform a partial fundoplication when we are operating on a patient with a distal diverticulum, with the choice of wrap being guided by the location of the diverticulum and the myotomy. We generally seek to cover the area of the myotomy with the wrap to bolster that tissue, which can be quite thin in patients who have mucosa that is chronically inflamed secondary to the diverticulum presence. Therefore, we would typically do a posterior Toupet partial fundoplication if the myotomy location is more posterior and an anterior Dor partial fundoplication if the myotomy location were more anterior.

The traditional approach to surgery had been to perform a thoracotomy (11), but thoracoscopic and laparoscopic approaches have been shown to be safe and effective with low morbidity (5-10). As described above, either laparoscopic or thoracoscopic approaches are feasible for distal diverticulum while a thoracoscopic approach is needed for more proximal diverticulum. For more proximal diverticulum, we approach them via right thoracoscopy. For very distal epiphrenic diverticulum, we prefer a laparoscopic approach that allows for a long myotomy to be extended onto the proximal stomach, and also allows for the performance of a concomitant fundoplication. In addition, we find that endoscopic staplers used to resect the diverticulum can be aligned more easily along the length of the esophagus for distal diverticulum in a laparoscopic approach by going from the peritoneal cavity into the posterior mediastinum through the hiatus. Lining up the stapler to go along the distal esophagus and towards the gastro-esophageal junction in a thoracoscopic approach is more awkward and technically difficult in our opinion.

Minimally invasive procedures can be accomplished with a low risk of conversion to open. The most serious risk of surgery is typically an esophageal leak, which has been estimated to occur in $14 \%$ of minimally invasive approaches (5). This leak rate tends to be a little bit higher than the rates reported in series of esophageal diverticulum managed via open techniques, which are $<10 \%$ in several series (11-14). However, the leak rate associated with minimally invasive surgery may improve with time, as some surgeons feel more leaks occurred early in the experience that led to revisions in techniques that have reduced the risk. We feel that the risk of leak can be mitigated with several intra-operative maneuvers and considerations. Careful handling of the esophagus is imperative, with the avoidance of excessive traction both on the esophagus in general and on the exposed esophageal mucosa at the neck of the diverticulum, which can be chronically inflamed and possibly somewhat friable and susceptible to tearing. Second, we recommend the use of staple loads designed for thin tissue such as vascular tissue rather than staple loads designed for thicker soft tissue. When the base of the 


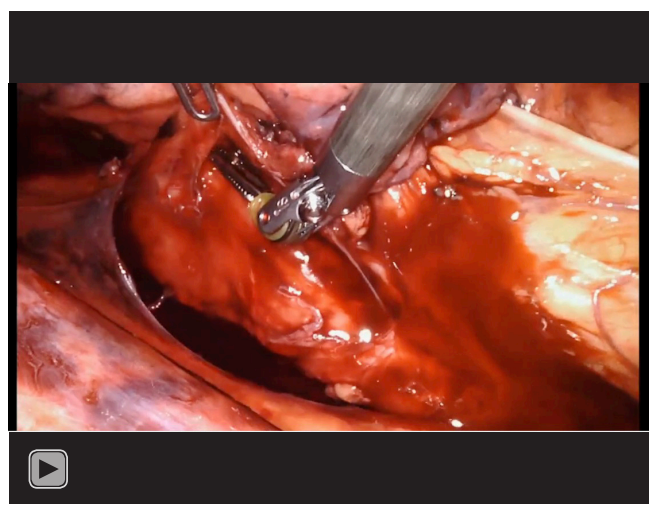

Video 1 Video depicting robotic-assisted esophageal diverticulectomy.

diverticulum has been appropriately mobilized, the stapler must only approximate two very thin layers of esophageal mucosa. Finally, we strongly recommend that intraoperative endoscopy be performed prior to completion of surgery so that any inadvertent injuries can be identified and repaired.

\section{Advantages of robotic-assisted thoracoscopic diverticulectomy}

The use of robotic-assisted technologies in thoracic surgery has been steadily increasing (15). As part of the armamentarium for minimally invasive approaches to the chest, robot-assisted platforms allow enhanced threedimensional visualization, wristed dexterity, and an improved learning curve. Modern robotic systems with improved docking capabilities have streamlined port placement and degrees of freedom leading to increased efficiency in robotic-assisted thoracoscopic procedures. Roboticassisted thoracoscopic surgery is safe with comparable morbidity, mortality, and complication rates as videoassisted thoracoscopic surgery. Furthermore, cosmesis and perioperative length of stay is equivalent to videoassisted procedures. Both minimally invasive approaches are associated with improved outcomes compared to open thoracotomy.

The exposure and resection of mid-esophageal diverticula is particularly more easily facilitated by a robot-assisted approach than a thoracoscopic approach. In our experience, we find that the visualization and wristed instrumentation of the robotic console allows for safe exposure of both the esophagus and the associated diverticulum. The use of the robot allows meticulous dissection of the posterior mediastinum, which allows safe mobilization of the esophagus. The dexterity of the robotic instruments allows both safe and expeditious performance of the procedure.

\section{Robotic esophageal diverticulectomy}

\section{Upper endoscopy}

The performance of the robotic esophageal diverticulectomy is demonstrated in the attached video (Video 1). The procedure is done after the induction of general anesthesia with the patient in the supine position. As discussed above, we recommend that endoscopy be performed at this point to re-evaluate the diverticulum, particularly if the surgeon has not already personally performed the endoscopy. In this case, endoscopy confirmed a normal proximal esophagus, and re-identified the location of the diverticulum. The large wide-mouthed diverticulum was located at $28-31 \mathrm{~cm}$ from the incisors. The patient still had significant debris within the diverticulum (Figure 4), which was emptied as much as possible so that debris would not be stapled into the mucosal closure at the time of the diverticulectomy later in the procedure. The more distal esophagus appeared normal. The gastro-esophageal junction was at $44 \mathrm{~cm}$, and the stomach appeared normal.

\section{Patient positioning}

After endoscopy is completed, lung isolation is achieved with either a bronchial blocker or a double-lumen endotracheal tube and the patient is placed in the left lateral decubitus position with the patient padded at all contact points. The operative table is flexed at the hips to open the intercostal spaces as much as possible, which allows ports to subsequently be placed with as minimal tension on the adjacent rib and intercostal nerve. This positioning not only makes port placement technically easier, but may also reduce both short-term and chronic patient pain after the procedure. The thorax should be in plane with the floor to allow easy docking of the robotic arms.

\section{Robotic port placement}

Figure 5 shows the port placement used for this procedure, which was performed with the Intuitive Si robot (Intuitive, Sunnyvale, CA, USA). An initial $8 \mathrm{~mm}$ robotic port was placed within the $7^{\text {th }}$ intercostal space at the anterior 


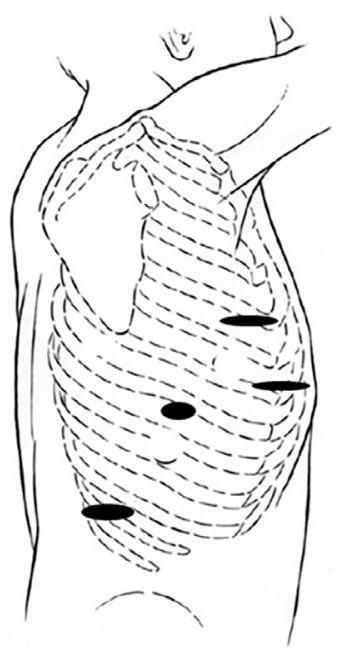

Figure 5 Robotic port placement for esophageal diverticulectomy.

axillary line. The chest was insufflated to $8 \mathrm{mmHg}$. Two additional $8 \mathrm{~mm}$ robotic ports placed anteromedially and posterolaterally $8 \mathrm{~cm}$ from the initially placed camera port. Following this, a $12 \mathrm{~mm}$ step port was introduced between the camera and the anterior-most robotic port. This port is used by the bedside assistant to pass instruments, aspirate fluid with a suction device, and provide additional retraction if needed. In addition, the assistant may pass an endoscopic stapler manually if the robotic stapling capability is not available. The robot is then docked.

\section{Initial esophageal mobilization}

After robot docking, the camera is installed followed by placement of a grasper on the right hand and a hook cautery on the left. We prefer the use of hook cautery to other instruments with energy capability, as we feel this tool minimizes possible inadvertent thermal injury to the underlying esophageal mucosa. The hemithorax is initially inspected and any lung adhesions are taken down. The inferior pulmonary ligament is then taken down with cautery to allow identification of the distal esophagus below the level of the inferior pulmonary vein, and then dissection is continued more superiorly posterior to the hilum of the right lung (Figure 6).

\section{Diverticulum mobilization and resection}

Further careful esophageal mobilization allows visualization of the diverticulum. In this case, a large diverticulum

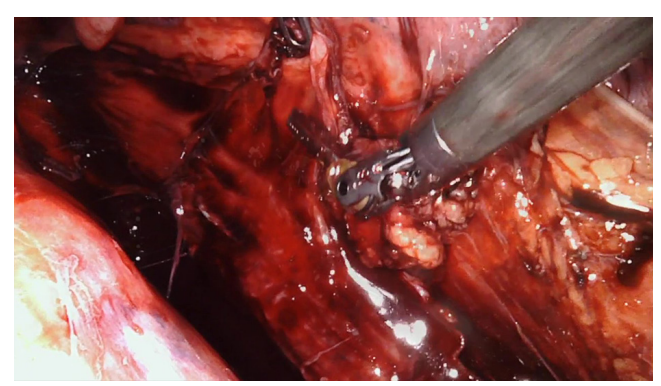

Figure 6 Dissection of the inferior pulmonary ligament and the mediastinum posterior to the hilum of the right lung exposes the esophagus in the posterior mediastinum.
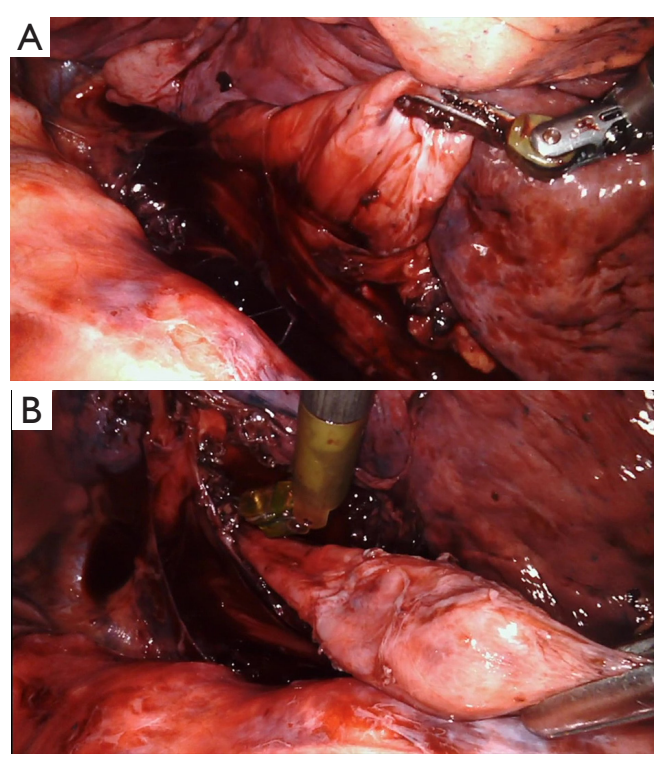

Figure 7 Further mobilization of the esophagus will (A) allow identification of the diverticulum, which should (B) be fully mobilized down to the neck of the diverticulum where it emerges from the otherwise grossly normal longitudinal esophagus.

was present as expected just above the level of the inferior pulmonary vein. After initial identification of the diverticulum, the diverticulum is then progressively mobilized and dissected out of the mediastinum anteriorly and posteriorly using both blunt dissection and cautery (Figure 7). Note that the esophageal diverticulum should be carefully but fully mobilized down to the neck of the diverticulum where it emerges from the more normal longitudinal esophagus. Grossly normal esophagus proximal and distal to the diverticulum should be exposed and visualized. This step is very important in preventing a 


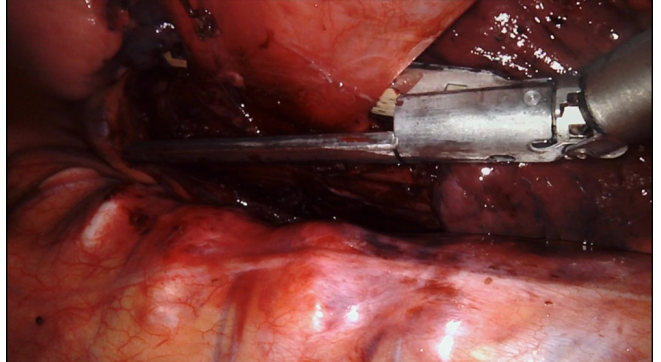

Figure 8 Diverticulectomy is accomplished by placing a linear stapler across the base of the esophageal diverticulum. The stapler is aligned along the length of the esophagus. We recommend that this part of the procedure be performed with an either an endoscope in place or a blunt bougie inside the esophagus to assess for and prevent inadvertent narrowing of the esophageal lumen.

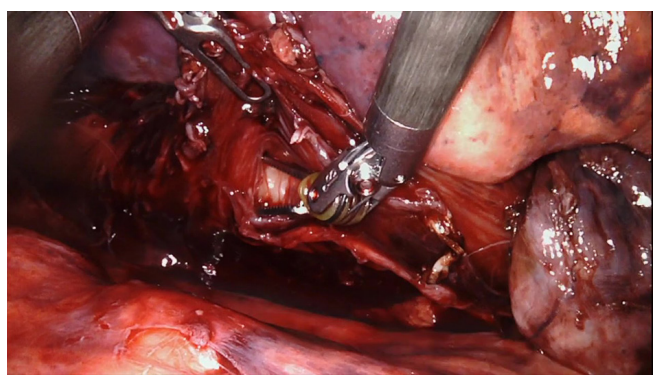

Figure 9 Esophageal myotomy opposite the location of the diverticulectomy is then performed both using the hook cautery instrument to divide first the longitudinal and then circular muscle layers. Blunt dissection with the grasper can also be used to expose the underlying esophageal mucosa, as demonstrated in this picture.

residual diverticulum from being present after resection. In this case, we repeated endoscopy at this point to assess for any inadvertent intraluminal injuries to the esophageal mucosa and also to ensure that all debris was cleared out of the diverticulum.

The assistant then placed an endo-GIA stapler (Medtronic, Minneapolis, MN, USA) with a vascular load was placed across the base of the diverticulum and closed (Figure 8). Endoscopy confirmed that the esophagus was not narrowed by the stapler placement, and firing of the stapler resected the diverticulum flush with the esophagus. The specimen was placed into a sterile protective bag, which was removed from the chest through the largest incision. Subsequent pathologic examination showed a segment of esophageal wall with acute and chronic inflammation consistent with diverticulum.
A bougie dilator can also be placed across the diverticulum to lessen the chance of narrowing the esophageal lumen when the diverticulum is resected. In our practice, we typically use a sized 48 to 54 French Hurst Dilator (Diversatek Healthcare, Milwaukee, WI, USA) with the specific size chosen based on the patient's size and body habitus (48 French for a small and thin patient and 54 French for a particularly large patient). The blunt tip of the Hurst dilator is preferred to possibly reduce the chance of inadvertent perforation compared to a dilator with an elongated tapered tip such as a Maloney dilator (Diversatek Healthcare). However, we selectively use the bougie for two reasons. First, the mucosa of the mobilized diverticulum can be extremely thin, and we do worry that for some patients that even a carefully placed blunt bougie could perforate through the diverticulum and substantially increase the complexity of the surgery. In addition, we also worry that there is an increased risk of inadvertent injury when manipulating the esophagus with the rigid bougie in place, particularly when the manipulation is being done via an instrument and not the surgeon's hand as during an open surgery. In cases where a bougie is not used, as in the case described, we place an adult sized endoscope so we can safely manipulate past the diverticulum. An adult sized endoscope is roughly 36 French in size, which allows an accurate estimate that the lumen is not being inadvertently significantly narrowed by the resection.

\section{Esophageal myotomy}

After diverticulum resection, the esophagus was inspected both via endoscopy and from inside the chest. The staple line was visually confirmed to appear intact, and no bubbling was noted from the staple line while insufflating the esophagus with the scope. A long esophageal myotomy is performed, approximately 180 degrees away from the diverticulum staple line. In this case, the location for the myotomy is also chosen at a site where the esophageal muscle had already been manipulated during the initial mobilization of the esophagus. The hook cautery instrument is used to divide the longitudinal and then the circular muscle layers. Blunt dissection with the grasper can also be used to expose the underlying esophageal mucosa (Figure 9). The myotomy can be then extended both proximally and distally to achieve whatever length of myotomy is desired. Saline can then be placed in the chest to cover the esophagus, and repeat endoscopy can be performed to again confirm complete resection of the diverticulum and 

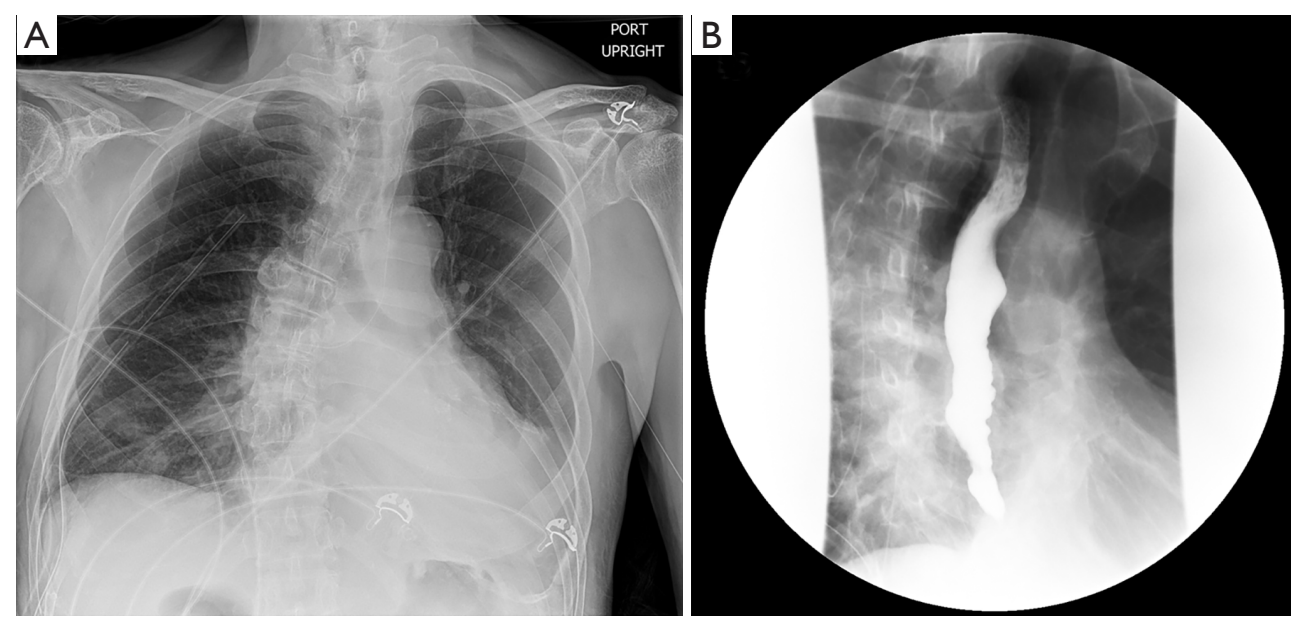

Figure 10 The (A) chest X-ray and (B) swallow study on post-operative day 1 following right thoracoscopy and robotic diverticulectomy of the patient with the esophageal diverticulum shown in Figure $2 \mathrm{~A}$.

also to make sure that there are no air bubbles in the chest secondary to leak or perforation while air was insufflated through the scope.

\section{Closure and post-operative care}

The robot is then un-docked, and a chest tube is placed through one of the port sites to lie posterior in the area of the esophageal manipulation. The incisions are closed after the lung is observed to fully re-inflate with ventilation by anesthesia. The patient is extubated in the operating room.

In this case, the patient appeared well in the morning after surgery with a clear chest X-ray (Figure 10A). A swallow study was then done on the first post-operative day that demonstrated complete diverticulum resection, no esophageal leak or stricture, and normal emptying of the esophagus (Figure 10B). The patient was started on a clear liquid diet that day. The chest tube was removed and the patient was discharged on post-operative day 2 on a full liquid diet. He was seen in clinic 3 weeks later and was doing well. He was advanced to a soft diet at that point and has had no further issues with dysphagia or regurgitation for over 4 years after surgery.

\section{Discussion}

Esophageal diverticula are relatively uncommon and can occur along any portion of the esophagus ${ }^{1}$. Diverticula in the distal aspect of the esophagus are typically false or pulsion diverticula that do not involve all layers of the esophageal wall $(2,3)$. These distal esophageal diverticula generally occur in the setting of motility disorders but can be found in patients who otherwise has normal esophageal motility. Larger esophageal diverticula are generally symptomatic, and intervention is typically indicated (3).

Surgical management of mid or distal esophageal diverticulum was traditionally done via a thoracotomy (11). Even when done by very experienced esophageal surgeons, these surgeries had a moderate risk for morbidity with post-operative esophageal leak rates of approximately $10 \%$ (11-14). Minimally invasive surgery via thoracoscopy or laparoscopy have recently been shown to be feasible and safe, though there was likely a learning curve to these procedures and complications such as leaks seemed to be more common early in surgeon's experiences (5-10).

In the case presented here, we have demonstrated a robotic-assisted thoracoscopic approach to management of a large mid-esophageal diverticulum. We feel that using the robot for this procedure greatly facilitates the performance of this procedure using minimally invasive technique. The improved visualization and instrument dexterity of the robot over a non-robotic thoracoscopic approach allows safe and relatively easy manipulation of both the normal esophagus as well as the diverticulum. The patient described in this case also had significant dextroscoliosis, which can sometimes make posterior mediastinal dissection more difficult due to altered anatomy. However, the visualization and instrument manipulation provided by the robot mitigated the effects of the scoliosis on the performance of the surgery. In our opinion, the robot is an ideal tool for 
surgically addressing relatively uncommon but potentially technically challenging esophageal diverticulum.

\section{Acknowledgment}

Funding: None.

\section{Footnote}

Provenance and Peer Review: This article was commissioned by the Guest Editor (Douglas Z. Liou) for the series "Advancement in Treatment for Esophageal Diseases" published in Journal of Visualized Surgery. This article has undergone external peer review.

Conflicts of Interest: Both authors have completed the ICMJE uniform disclosure form (available at https://jovs. amegroups.com/article/view/10.21037/jovs-2019-27/coif). The series "Advancement in Treatment for Esophageal Diseases" was commissioned by the editorial office without any funding or sponsorship. The authors have no other conflicts of interest to declare.

Ethical Statement: The authors are accountable for all aspects of the work in ensuring that questions related to the accuracy or integrity of any part of the work are appropriately investigated and resolved. The study was conducted in accordance with the Declaration of Helsinki (as revised in 2013). Our Institution's IRB does not require approval for case reports, but informed consent was obtained from the patient whose case was described and presented.

Open Access Statement: This is an Open Access article distributed in accordance with the Creative Commons Attribution-NonCommercial-NoDerivs 4.0 International License (CC BY-NC-ND 4.0), which permits the noncommercial replication and distribution of the article with the strict proviso that no changes or edits are made and the original work is properly cited (including links to both the formal publication through the relevant DOI and the license). See: https://creativecommons.org/licenses/by-nc-nd/4.0/.

\section{References}

1. Smith CD. Esophageal strictures and diverticula. Surg Clin North Am 2015;95:669-81.
2. Law R, Katzka DA, Baron TH. Zenker's diverticulum. Clin Gastroenterol Hepatol 2014;12:1773-82; quiz e111-2.

3. Herbella FA, Patti MG. Modern pathophysiology and treatment of esophageal diverticula. Langenbecks Arch Surg 2012;397:29-35.

4. Ishaq S, Sultan H, Siau K, et al. New and emerging techniques for endoscopic treatment of Zenker's diverticulum: state-of-the-art review. Dig Endosc 2018;30:449-60.

5. Kilic A, Schuchert MJ, Awais O, et al. Surgical management of epiphrenic diverticula in the minimally invasive era. JSLS 2009;13:160-4.

6. Galata CL, Bruns CJ, Pratschke S, et al. Thoracoscopic resection of a giant midesophageal diverticulum. Ann Thorac Surg 2012;94:293-5.

7. Soares RV, Montenovo M, Pellegrini CA, et al. Laparoscopy as the initial approach for epiphrenic diverticula. Surg Endosc 2011;25:3740-6.

8. Isaacs KE, Graham SA, Berney CR. Laparoscopic transhiatal approach for resection of midesophageal diverticula. Ann Thorac Surg 2012;94:e17-9.

9. van der Peet DL, Klinkenberg-Knol EC, Berends FJ, et al. Epiphrenic diverticula: minimal invasive approach and repair in five patients. Dis Esophagus 2001;14:60-2.

10. Fernando HC, Luketich JD, Samphire J, et al. Minimally invasive operation for esophageal diverticula. Ann Thorac Surg 2005;80:2076-80.

11. Varghese TK Jr, Marshall B, Chang AC, et al. Surgical treatment of epiphrenic diverticula: a 30-year experience. Ann Thorac Surg 2007;84:1801-9; discussion 1801-9.

12. Benacci JC, Deschamps C, Trastek VF, et al. Epiphrenic diverticulum: results of surgical treatment. Ann Thorac Surg 1993;55:1109-13; discussion 1114.

13. Streitz JM Jr, Glick ME, Ellis FH Jr. Selective use of myotomy for treatment of epiphrenic diverticula. Manometric and clinical analysis. Arch Surg 1992 ;127:585-7; discussion 587-8.

14. Nehra D, Lord RV, DeMeester TR, et al. Physiologic basis for the treatment of epiphrenic diverticulum. Ann Surg 2002;235:346-54.

15. Marshall MB, Wee JO. Robotic platform use in general thoracic surgery. JAMA Surg 2019;154:1066-7.

doi: 10.21037/jovs-2019-27

Cite this article as: Rosenberg G, Berry MF. Robotic-assisted esophageal diverticulectomy. J Vis Surg 2021;7:16. 